\title{
Absenteísmo em Hospital Filantrópico de Médio Porte
}

\section{Absenteeism at a Medium Size Philanthropic Hospital}

\author{
Roseli Landgraf Fernandes ${ }^{1}$; Maria do Carmo Lourenço Haddad²; Adriana Estela \\ Pinesso Morais ${ }^{3}$; Ilidia Terezinha Martelli Takahashi ${ }^{4}$
}

\begin{abstract}
Resumo
O absenteísmo é um complexo problema administrativo para as organizações. Nos hospitais, tornase ainda mais complicado, pois o cuidado ao cliente não pode ser interrompido. Entende-se por absenteísmo-doença, as faltas ao trabalho por problemas de saúde. Tem-se como objetivo analisar as faltas dos trabalhadores por causas de saúde relacionando-as com idade, sexo, tempo de trabalho na instituição, turno de trabalho e categoria funcional. Trata-se de um estudo retrospectivo, descritivo e documental. Os dados foram coletados dos atestados de saúde apresentados no período de 01 julho de 2007 a 30 de junho de 2008 ao setor de recursos humanos de um hospital filantrópico de médio porte. As análises dos resultados demonstraram que o número de atestados apresentados é maior no sexo feminino $(90,80 \%)$ e em funcionários com menor tempo de trabalho na instituição $(61,19 \%)$. Verificou-se que o número de atestados é mais elevado nos trabalhadores que fazem revezamento entre os turnos matutino e vespertino. Também foi observado que os trabalhadores com idade acima de 48 anos, apresentaram menor número de atestados que os mais jovens. Os auxiliares e técnicos de enfermagem foram as categorias profissionais que mais apresentaram atestados (46,05\%). Os dados encontrados permitem identificar vários fatores relacionados ao absenteísmo e fornecem subsídios para reforçar ações que acompanhem as questões de saúde dos trabalhadores e também a implantação de ações que visem à melhoria da qualidade de vida no trabalho.
\end{abstract}

Palavras chave: Absenteísmo. Atestado de saúde. Trabalhador. Hospital médio porte.

\begin{abstract}
The absenteeism is a complex administrative problem for organizations. In hospitals, it becomes even more complicated as the patient care can not be interrupted. The term absenteeism - disease, refers to the time off work due to health problems. It is intended to analyze the absence of workers due to health problems is related to age, sex, length of employment at the institution, part work shift and functional category. This is a retrospective, descriptive and documentary study. Data was collected from medical certificates submitted in the period from 01 July 2007 to 30 June 2008 to the sector of human resources of a medium-sized philanthropic hospital. The analysis results show that the number of certificates is greater in females $(90.80 \%)$ and staff that work less time in the institution $(61.19 \%)$. It was found that the number of certificates is higher in workers who make the rotation between morning and afternoon shifts. It was also noted that workers aged over 48 years, had lower number of certificates than the younger ones. The nursing technicians assistant were the professional categories that had presented more certificates $(46.05 \%)$. The results identify several factors related to absenteeism and provide subsidies to strengthen actions to monitor the health of workers and also the implementation of actions
\end{abstract}

\footnotetext{
1 Nutricionista chefe do serviço de nutrição da Casa de Misericórdia de Cornélio Procópio, especialista em Nutrição Clinica e em Administração Hospitalar. Av. Nossa Senhora do Rocio, 1165, Centro, Cornélio Procópio, PR, 86300-000. Email: nutri-roseli@ uol.com.br.

$2 \mathrm{Dr}^{\mathrm{a}}$ em Enfermagem, docente do curso de enfermagem da UEL, PR

Enfermeira chefe da Seção Materno-Infantil do Ambulatório do Hospital de Clínicas, HU/UEL

${ }^{4}$ Nutricionista chefe da Seção de Nutrição do Ambulatório do Hospital de Clínicas, HU/UEL
} 
aimed at improving the quality of life at work.

Keywords: Absenteeism. Certificate of health. Worker. Midsize hospital.

\section{Introdução}

A palavra "absenteísmo" tem sua origem no francês absentéisme e significa falta de assiduidade ao trabalho ou a outras obrigações sociais (FERREIRA, 1999). Segundo Quick e Lapertosa (1982), o absenteísmo é dividido em absenteísmo voluntário (ausência no trabalho por razões particulares); absenteísmo por doença (inclui todas as ausências por doença ou por procedimento médico, excetuam-se os infortúnios profissionais); absenteísmo por patologia profissional (ausências por acidentes de trabalho ou doença profissional); absenteísmo legal (faltas no serviço amparadas por leis, tais como: gestação, nojo, gala, doação de sangue e serviço militar) e absenteísmo compulsório (impedimento ao trabalho devido a suspensão imposta pelo patrão, por prisão ou outro impedimento que não permita o trabalhador chegar ao local de trabalho).

Em hospitais, o absenteísmo tem recebido destaque nos últimos anos, pois o caráter penoso deste trabalho é influenciado pela grande quantidade de atividades que exigem esforço físico, por más condições do ambiente de trabalho e por tensões nas relações interpessoais. Nesse contexto, o trabalho em hospitais pode ser um agente catalisador do processo de desgaste do trabalhador, culminando em doenças (REIS, 2003).

A maioria das ausências são justificadas por atestados médicos, o que não significa que todas as ausências sejam sempre decorrentes de causas médicas e que a etiologia do absenteísmo é multifatorial, dependendo da sua origem que pode ser decorrente de fatores da atividade laboral, perilaborais, do meio extralaboral, patologias sofridas pelo trabalhador, fatores individuais e fatores dependentes do sistema administrativo (OTERO, 1993). O absenteísmo é um dos problemas administrativos mais onerosos para qualquer instituição.

O trabalho tem papel fundamental na inserção dos indivíduos no mundo e contribui para a formação de sua identidade, entretanto, na forma em que o trabalho está organizado e é executado por um grande contingente de trabalhadores, na sociedade atual, predominam seus efeitos negativos, o adoecimento e a morte (CODO; SAMPAIO; HITOMI, 1992).

A maior preocupação do setor de saúde ocupacional de qualquer empresa está relacionada com a saúde do trabalhador. Segundo a Organização Mundial da Saúde (1948 apud GONTIJO, 2005) "saúde é o completo bem estar físico, mental e social e não apenas a ausência de doença" (BRASIL, 1998 apud GONTIJO, 2005). De acordo com Rodrigues (2004), a saúde é resultante das condições de alimentação, habitação, educação, renda, meio ambiente, trabalho, transporte, emprego, lazer, liberdade, acesso e posse de terra e aos serviços de saúde.

A insatisfação do indivíduo com a sobrecarga de trabalho devido à falta de pessoal e condições inadequadas na infra-estrutura da instituição pode contribuir para elevar o índice de absenteísmo, desencadeando a queda da qualidade do cuidado prestado ao homem.

Silva e Marziale (2000) afirmam que não parece existir uma relação precisa de causa e efeito, mas sim, um conjunto de variáveis que podem levar ao absenteísmo como fatores do trabalho, sociais, fatores culturais, de personalidade e de doenças.

As autoras ressaltam que as causas do absenteísmo nem sempre estão no trabalhador, mas na empresa, enquanto organização e 
supervisão deficientes, por meio da repetitividade de tarefas, da desmotivação e desestímulo, das condições desfavoráveis do ambiente e do trabalho, da precária integração entre os empregados e a organização, bem como dos impactos psicológicos de uma direção deficiente, que não visa à uma política prevencionista e humanista.

Os riscos para a saúde relacionados com o trabalho dependem do tipo de atividade profissional e das condições em que a mesma é desempenhada (GASPAR, 1997). O autor complementa que os serviços de saúde em particular os hospitais proporcionam aos seus trabalhadores condições de trabalho reconhecidamente piores em relação aos demais serviços de saúde.

Os administradores em geral devem ter a preocupação de prever e prover os recursos humanos em número e competência para o desenvolvimento das atividades necessárias para o cumprimento dos objetivos organizacionais e da qualidade da assistência prestada (PARRA, 2003).

Devido ao fato do absenteísmo ser um problema no gerenciamento dos recursos humanos na Instituição onde este estudo foi realizado, tem-se como objetivo analisar os atestados de saúde apresentados por trabalhadores de uma instituição hospitalar de médio porte.

\section{Material e métodos}

Trata-se de pesquisa descritiva quantitativa e documental retrospectiva, realizada na Casa de Misericórdia de Cornélio Procópio, um hospital filantrópico de médio porte, localizada na região Norte do Paraná, que possui 139 leitos. É referência para atendimento da $18^{\mathrm{a}}$ Regional de Saúde do Paraná, que abrange 21 municípios, os quais somam aproximadamente 227.000 habitantes. O Corpo Clínico é formado por 70 profissionais médicos e possui 311 servidores distribuídos em duas gerências de prestação de serviços, sendo a de enfermagem constituída por 155 funcionários e a administrativa com 156 funcionários.

Os trabalhadores são contratados sob o regime da Consolidação das Leis de Trabalho (CLT), sendo selecionados pela própria equipe de recursos humanos da Instituição. O regime de trabalho da equipe de enfermagem é de 36 horas semanais, distribuídos em escala de revezamento em turno de 6 horas em semanas alternadas manhã e tarde e o turno da noite de 12 horas de trabalho por 36 horas de descanso. A equipe administrativa trabalha 44 horas semanais sendo 8 horas diárias de segunda a sexta-feira e 4 horas no sábado.

Os dados foram coletados dos atestados de saúde apresentados ao serviço de recursos humanos da instituição, no período de 01 julho de 2007 a 30 de junho de 2008, utilizando um instrumento pré-elaborado (Apêndice A).

Não foram incluídos neste estudo os atestados de acompanhamento de familiares, considerando a Convenção Coletiva do Sindicato de Saúde, que determina que estes atestados justificam ausências, mas não abonam faltas.

Os afastamentos foram computados nos seguintes intervalos: de 1, 2 e 3 dias, de 4 a 15 , e superior a 15 dias. No contexto legal, para afastamento superior a 15 dias, o trabalhador necessita ser submetido à perícia médica.

$\mathrm{Na}$ análise dos atestados médicos, foram considerados, o tempo de afastamento e a especialidade médica. Deve-se destacar que os atestados com até 15 dias são arquivados no setor de recursos humanos da instituição e, por lei, não há obrigatoriedade da identificação do diagnóstico médico. Os diagnósticos dos afastamentos superiores há 15 dias foram registrados de acordo com os grandes grupos da $10^{\mathrm{a}}$ Revisão da Classificação Internacional de 
Doenças (2003).

Outras variáveis relacionadas ao funcionário que pudessem estar associadas aos afastamentos foram analisadas: sexo, idade, categoria funcional, tempo de serviço na instituição, jornada e turno de trabalho.

Os dados quantitativos foram agrupados e organizados em um banco de dados, com o auxílio do Programa Epi Info 2003, análise descritiva SPSS 13.0, organização e formatação em tabelas pelo Word e Excel 2003.

Anterior à coleta dos dados, foi solicitada autorização por escrito da Direção da instituição para a realização da pesquisa o que foi autorizado e o documento encontra-se arquivado com os autores.

\section{Resultados}

Os dados da Tabela 1 demonstram que dos 311 funcionários, 152 apresentaram atestados no período estudado, representando $48,87 \%$ do total, sendo que $50,70 \%$ dos atestados apresentados eram de trabalhadores da área de enfermagem e $49,30 \%$ da área administrativa. Considerando que o total de funcionários da equipe de enfermagem é de 155 trabalhadores, ou seja, 49,83\% do total de funcionários do hospital, não houve diferença significativa entre os atestados apresentados pelos funcionários da área administrativa, que somam 156 trabalhadores (50,17\%). Quanto ao sexo dos profissionais, $90,80 \%$ eram do sexo feminino e $9,20 \%$ eram do sexo masculino (Tabela 1). Considerando que dos 311de trabalhadores, 275 são mulheres e 36 são homens, observou-se que $50,18 \%$ das mulheres e $38,88 \%$ dos homens em algum período se ausentaram do trabalho.

Em relação à idade dos trabalhadores que apresentaram atestados 30,92\% tinham idade entre 28 a 37 anos, $22,37 \%$ entre 48 a 57 anos, $21,71 \%$ entre 18 a 27 anos, $18,42 \%$ entre 38 e 47 anos e $6,58 \%$ idade acima de 58 anos.

Quanto à categoria funcional, 50,70\% eram da área de enfermagem, sendo 5,26\% enfermeiros e $46,05 \%$ auxiliares e técnicos de enfermagem, $13,81 \%$ eram copeiras e auxiliares de cozinha, $11,84 \%$ eram auxiliares de escritório, secretárias e $23,86 \%$ englobaram as demais categorias. Observou-se que $64,50 \%$ dos funcionários que apresentaram atestados de saúde, faziam jornada de 36 horas semanais, 34,20\% jornada de 44 horas semanais e 1,30\% 20 horas semanais. Com relação ao turno de trabalho, $44,10 \%$ dos funcionários trabalhavam em esquema de revezamento entre o período matutino e vespertino, $31,60 \%$ trabalhavam no período diurno, $23,00 \%$ no noturno e $1,30 \%$ no período vespertino.

Quanto ao tempo de trabalho na instituição, $66,45 \%$ dos funcionários trabalhavam entre $1 \mathrm{e}$ 9 anos, 33,55\% trabalhavam no hospital há 10 anos ou mais. 
Tabela 1 - Distribuição dos servidores por gerência, sexo, idade, categoria profissional, jornada de trabalho semanal, turno e tempo de trabalho de funcionários que apresentaram atestado de saúde em hospital Filantrópico de médio porte.

\begin{tabular}{lll}
\hline Variáveis & $\mathbf{N}^{\mathbf{0}}$ & $\%$ \\
\hline Gerência
\end{tabular}

\section{Gerência}

Administrativa

75

49,30

Enfermagem

50,70

\section{Sexo}

Feminino

Masculino

\section{Idade}

De 18 a 27 anos

De 28 a 37 anos

De 38 a 47 anos

De 48 a 57 anos

De 58 a 67 anos

Acima de 67 anos

\section{Categoria Profissional}

Enfermeiro, Auxiliar e Técnico de Enfermagem 77

Copeira e Auxiliar de Cozinha

Auxiliar de Escritório, Secretária, Recepcionista

Outros

\section{Jornada de Trabalho Semanal}

20 horas semanais

36 horas semanais

44 horas semanais

Integral

Manhã

Manhã/Tarde

Noite

\section{Tempo de trabalho}

Até 3 anos

De 4 a 6 anos

De 7 a 9 anos

8

5,26

10 anos ou mais 
Em relação aos atestados apresentados que totalizaram 286 , verificou-se que $47,90 \%$ eram para afastamento de 1 dia, 15,73\% para afastamento de 2 dias, 8,39\%, para afastamento de 3 dias, 23,08\% para afastamento de 4 a15 dias, e 4,90\% acima de 15 dias (Tabela 2).

Quanto às especialidades médicas verificadas no atestado de saúde, $65,38 \%$ dos trabalhadores foram atendidos no pronto-socorro, $8,39 \%$ em ginecologia e obstetrícia, 4,20\% em neurologia, $3,85 \%$ em ortopedia e $3,50 \%$ em urologia. Outras especialidades foram observadas em $8,39 \%$ dos atestados. Também foi verificado que $6,29 \%$ dos trabalhadores apresentaram atestados para atendimento em serviço de odontologia.

Tabela 2 - Distribuição do tempo de afastamento e especialidade que forneceu os atestados de saúde apresentados por funcionários de um hospital filantrópico de médio porte.

\begin{tabular}{lll}
\hline Variáveis & $\mathbf{N}^{\mathbf{0}}$ & $\mathbf{\%}$ \\
\hline Dias & 137 & 47,90 \\
1 dia & 45 & 15,73 \\
2 dias & 24 & 8,39 \\
3 dias & 66 & 23,08 \\
De 4 a 15 dias & 14 & 4,90 \\
Acima de 15 dias & & \\
Especialidade & 187 & 65,38 \\
Pronto-Socorro & 24 & 8,39 \\
Ginecologista e Obstetrícia & 18 & 6,29 \\
Odontologia & 12 & 4,20 \\
Neurologia & 11 & 3,85 \\
Ortopedia & 10 & 3,50 \\
Urologia e Nefrologia & 24 & 8,39 \\
Outros & 286 & 100 \\
\hline Total & & \\
\hline
\end{tabular}

Em relação aos diagnósticos médicos citados nos atestados para afastamento de mais de 15 dias, verificou-se que $28,57 \%$ foram de episódios depressivos, 14,28\% de distensão da articulação do joelho. Os demais CID foram registrados em apenas um atestado e estão apresentados na Tabela 3. 
Tabela 3 - Distribuição dos diagnósticos médicos descritos nos atestados de saúde apresentados por funcionários de um hospital filantrópico de médio porte.

\begin{tabular}{llc}
\hline CID & $\mathbf{N}^{\mathbf{0}}$ & $\mathbf{\%}$ \\
\hline Episódios depressivos & 4 & 28,57 \\
Distensão do joelho & 2 & 14,29 \\
Cólica renal & 1 & 7,14 \\
Fratura de extremidade de úmero & 1 & 7,14 \\
Hérnia inguinal & 1 & 7,14 \\
Hipertensão arterial & 1 & 7,14 \\
Intoxicação por antipsicóticos e psicodislépticos & 1 & 7,14 \\
Sintomas de algias cefálicas & 1 & 7,14 \\
Tenossinovites & 1 & 7,14 \\
Traumatismo do tornozelo ou pé & 1 & 7,14 \\
\hline Total & 14 & 100 \\
\hline
\end{tabular}

\section{Discussão}

Analisando os dados encontrados, observouse que não houve diferença significativa entre o número de funcionários que apresentaram atestados da área de enfermagem em relação aos da área administrativa. Porém, quando se observa o número de funcionários que apresentaram atestados por categoria funcional, encontram-se os auxiliares e técnicos de enfermagem como a categoria mais prevalente.

Algumas atividades executadas por trabalhadores de enfermagem provocam demasiado desgaste físico, tais como o transporte, a movimentação de equipamentos e de pacientes, a longa permanência em pé durante a assistência, entre outras atividades, associados a má postura corporal, inadequação do espaço físico e mobiliário, são fatores de risco ergonômico responsáveis por danos à saúde (MENDES, 1995).

As condições de trabalho da equipe de enfermagem implicam longas jornadas no trabalho em turnos desgastantes (vespertino e noturno, domingos e feriados), nos rodízios, em multiplicidade de funções, repetitividade, monotonia, intensividade e ritmo excessivo de atividades, ansiedade, esforço físico, posições incômodas, na separação do trabalho intelectual e manual, no controle das chefias, desencadeando acidentes e doenças (PITTA, 2003).

Observou-se que 50,32\% dos funcionários da equipe de enfermagem tiveram assiduidade no período estudado, índice muito relevante quando comparado com estudo de Silva e Marziale (2003), no qual o índice de assiduidade foi de $25 \%$.

Estudos predizem que taxas de incapacidade laboral são reduzidas pelo medo de dispensa do trabalho que é diferente dos estatutários que se sentem mais seguros ao se afastarem (SILVA; MARZIALE, 2003).

Observou-se que houve elevado absenteísmo no setor de nutrição e dietética, indicando a necessidade da realização de um estudo específico para esta área, considerando os determinantes do processo saúdedoença a que estes trabalhadores estão submetidos.

Em relação à predominância de sexo feminino na apresentação de atestados de saúde, é possível que uma maior proporção de ausência esteja relacionada à dupla jornada que na maioria das 
vezes, não é caracterizada por dois empregos, mas pelo trabalho e afazeres domésticos que as mulheres realizam após a jornada normal. Características de morbidade feminina, como os distúrbios ligados ao ciclo hormonal, também são determinantes do absenteísmo entre as mulheres (ROBBINS, 2005).

As mulheres trazem consigo outros problemas que não somente os seus pessoais, mas dos filhos, marido, como doenças, reuniões na escola e outras atividades, que levam muitas vezes essa mulher a faltar no serviço. Esses motivos não aparecem nas justificativas dos homens, que não se sentem na obrigação de realizá-las (GEDVILA, 2006).

Em relação à idade dos profissionais, Reis (2003), Barbosa e Soler (2003) encontraram maior absenteísmo em idades mais jovens. Já Inoue et al. (2008) e Frutuoso e Cruz (2005) encontraram maior frequência de afastamentos na faixa etária acima de 40 anos.

No presente trabalho, verificou-se que os trabalhadores na faixa etária entre 18 a 37 anos apresentaram maior número de atestados de saúde, provavelmente devido ao ritmo de trabalho intenso, vida familiar com filhos na infância e possibilidade de duplo vínculo de trabalho, frequentemente presente nessa faixa etária, o que pode ter influenciado em maior absenteísmo.

Este fato também pode estar relacionado ao turno de trabalho, no qual se verificou maior absenteísmo no turno de revezamento entre matutino e vespertino, para o grupo de funcionários com carga horária de 36 horas semanais.

Os fatores relacionados ao tempo e ao ritmo de trabalho são muito importantes na determinação do sofrimento psíquico relacionado ao trabalho. Jornadas de trabalho longas, com poucas pausas destinadas ao descanso e/ou refeições de curta duração, em lugares desconfortáveis, turnos de trabalho noturnos, turnos alternados ou turnos iniciando muito cedo pela manhã, ritmos intensos ou monótonos, submissão do trabalhador ao ritmo de máquinas, pressão de supervisores e chefes por mais velocidade, causam com frequência, quadros ansiosos, fadiga e distúrbios do sono (BRASIL, 2001).

Conforme afirma Rotemberg et al. (2001), a idade geralmente está associada à instabilidade de ritmos circadianos, distúrbios do sono, depressão e um declínio na capacidade física e na saúde, favorecendo, portanto, a uma intolerância progressiva por parte do trabalhador.

Os autores Koller (1983) e Haider et al. (1988) descrevem que quanto maior o número de anos trabalhando em turnos diferenciados, maior o número de queixas e o desenvolvimento de patologias associadas a esse tipo de esquema de trabalho.

À medida que as pessoas envelhecem, modificam certas características dos ritmos biológicos que estão de certa forma associadas à tolerância ao trabalho em turnos. Moreno, Fischer e Rotenberg (2003) chamam a atenção para esse problema, pois haverá maiores contingentes de trabalhadores em turnos com idades próximas aos 50 anos ou mais, no final dessa década. Essas pessoas, valiosas para as empresas nas quais trabalham, poderão enfrentar mais dificuldades no trabalho em turnos, especialmente se as jornadas de trabalho forem mais extensas (12 horas diárias) e tiverem menos folgas semanais.

Em relação ao tempo de trabalho na instituição, $61,19 \%$ dos atestados apresentados eram de servidores que trabalhavam no hospital entre 1 a 6 anos.

Quanto ao período de afastamento, observouse que a $47,90 \%$ dos atestados apresentados foi de afastamento de curta duração, ou seja, de um dia. Em estudo realizado por Bittencourt (1993), verificouse que a categoria profissional que apresentou maior número de atestados de 1, 2 ou 3 dias por doença foi dos trabalhadores de enfermagem, e $74,2 \%$ afastaram-se até 15 dias e $25,8 \%$ permaneceram ausentes de suas atividades por um período superior a 15 dias. 
Em relação à especialidade médica mais referida nos atestados médicos, 187 foram do pronto socorro, o que foi relacionado ao fato do hospital oferecer aos seus funcionários prioridade de atendimento no próprio pronto socorro, tornando o acesso o mais ágil e rápido, garantindo inclusive a internação gratuita em caso de necessidade.

Como não é exigido o registro do CID nos atestados para afastamento inferiores a 15 dias, somente foi possível avaliar os diagnósticos dos atestados de mais de 15 dias que somam 14, onde foram observados $28,57 \%$ de episódios depressivos e em segundo lugar, distensão de joelho com $14,29 \%$.

A intensificação laboral é traço característico da atual fase do capitalismo e tem levado ao consumo desmedido das energias físicas e espirituais dos trabalhadores (NAVARRO; PADILHA, 2007). Nesse contexto de alta demanda por serviços e exigências, provavelmente aumente a taxa de absenteísmo por doença.

\section{Considerações Finais}

Um hospital, pelas peculiaridades de trabalho do dia a dia apresenta grande complexidade em administrar pessoas. O ambiente é de tensão emocional contínua, envolvendo pacientes, familiares e visitas num confronto diário de dor e sofrimento. Os horários de trabalho são inadequados, pois o hospital tem de funcionar 24 horas por dia. Os salários nem sempre são compatíveis com essas exigências e com o nível de tensão vivenciado. As margens de lucro de um hospital filantrópico são irrisórias e quase nunca se investe em salários de funcionários, e sim em estruturas e equipamentos para o trabalho.

A análise dos resultados demonstrou que os funcionários com menor tempo de trabalho na instituição tiveram maior número de atestados, o que sugere a necessidade de elaborar estratégias de recepção e acolhimento do novo funcionário para propiciar um envolvimento do mesmo com a instituição.

Observou-se também que os funcionários mais jovens (18 a 37 anos de idade) apresentaram maior número de atestados. Portanto torna-se necessário uma investigação aprofundada, a fim de verificar os motivos dos atestados e suas relações com a sobrecarga de trabalho, duplos vínculos empregatício, falta de envolvimento do trabalhador ou gerência, baixa satisfação, entre outros.

Neste estudo, o número de trabalhador da área administrativa que apresentou atestados foi praticamente igual ao da área de enfermagem, fato que provoca necessidade de estudos posteriores considerando que a maioria dos estudos realizados contempla apenas a área de enfermagem.

Verificou-se que o número de atestados para afastamento de um dia e fornecido por profissionais do pronto-socorro da própria instituição é elevado, sendo necessário rever a estratégia de atendimento médico aos funcionários para melhorar $\mathrm{o}$ acompanhamento da saúde do trabalhador.

Concluiu-se que, nos intervalos de 4 a 15 dias, o número de atestados apresentados é maior do que nos superiores a 15 dias, provavelmente porque as instituições privadas arcam com o ônus do trabalhador afastado por doença neste período e acima de 15 dias de licença, os benefícios são pagos pelo Governo que, por vezes parcela ou atrasa no pagamento do benefício, interferindo na vida pessoal do indivíduo.

No período analisado observou-se que os atestados apresentados superiores a 15 dias foram associados a trabalhadores com dedicação de 10 anos ou mais na instituição, deduzindo que exista maior envolvimento dessas pessoas com o local e grupo de trabalho, levando o trabalhador a ausentarse apenas por patologia grave que o incapacite às suas atividades, o que diferencia dos funcionários com menos de 6 anos na instituição, que apresentam grande numero de atestados de um a três dias, principalmente de consultas eletivas ou até mesmo 
de pronto socorro, para cuidados de familiares.

Destaca-se o elevado número de atestados apresentados pela equipe de enfermagem que trabalha em turno de revezamento entre manhã e tarde, indicando a necessidade de análise aprofundada desta escala de trabalho.

Concluiu-se que a não obrigatoriedade de registro do CID impossibilita o estudo das causas do afastamento, dificultando ações preventivas específicas.

Verificou-se que a instituição tem trabalhado na busca de um relacionamento interpessoal satisfatório, exemplo disso são as atividades sociais desenvolvidas, como a festa do trabalhador, semana da saúde, a festa de confraternização do Natal, homenagem aos funcionários que completam 30 anos de empresa, cesta de alimentos fornecida trimestralmente e ainda o abono salarial, conforme produtividade e assiduidade no decorrer do ano.

A saúde e a qualidade de vida dos trabalhadores interferem na qualidade do trabalho, portanto é necessário que os gestores acompanhem e propiciem condições adequadas para execução das atividades com foco na manutenção da saúde dos trabalhadores.

\section{REFERÊNCIAS}

BARBOSA, D. B.; SOLER, Z. A. S. G. Nursing absenteeism: occurences at a university hospital. Revista Latino Americana de Enfermagem, São Paulo, v. 11, n. 2, p. 177-183, 2003.

BITTENCOURT, C. M. Doenças do trabalho e o exercício da enfermagem. 1993. Dissertação (Mestrado em Enfermagem) - Escola de Enfermagem da Salvador, Universidade Federal da Bahia.

BRASIL. Ministério da Saúde. Doenças relacionadas ao trabalho: manual de procedimentos para os serviços de saúde. Brasília: Ministério da Saúde, 2001. (Série A. Normas e Manuais Técnicos, n. 114).

ORGANIZAÇÃO MUNDIAL DE SAÚDE. Classificação internacional de doenças. 10. ed. São Paulo: Ed. Universidade de São Paulo, 2003.
CODO, W.; SAMPAIO, J. J. C.; HITOMI, A. H. Indivíduo, trabalho e sofrimento: uma abordagem interdisciplinar. Petrópolis: Vozes, 1992.

FERREIRA, A. B. H. Novo dicionário da língua portuguesa. 3. ed. Rio de Janeiro: Nova Fronteira, 1999.

FRUTUOSO, J. T.; CRUZ, R. M. Mensuração da carga de trabalho e sua relação com a saúde do trabalhador. Revista Brasileira de Medicina do Trabalho, São Paulo, v. 3, n. 1, p. 29-36, jan./jul. 2005.

GASPAR, P. J. S. Enfermagem profissão de risco e de desgaste: perspectivas do enfermeiro do serviço de urgência. Nursing, Lisboa, v. 109, n. 10, p. 24, mar. 1997.

GEDVILA, M. T. L. A enfermagem que cuida, também adoece: uma revisão integrativa. 2006. Monografia (Especialização em Enfermagem do Trabalho) - Centro Universitário Filadélfia, Londrina. 2006.

GONTIJO, E. O que é saúde? Disponível em: <http:/ www.cdof.com.br/consult45.htm>. Acesso em: 25 jul. 2005.

HAIDER, M. et al. A destabelizacion theory of shiftwork effects. In: HEKKENS, W. TH. J. M.; KERFHORK, G.; RIETVELD, W. J. (Ed.). Thendes in Chronobiology. Oxford: Pergamon Press, 1988. p. 209-217.

INOUE, K. C.; MATSUDA, L. M.; SILVA, D. M. P. P.; UCHIMURA, T. T.; MATHIAS,T. A. F. Absenteísmodoença da equipe de enfermagem em unidade de terapia intensiva. Revista Brasileira de Enfermagem, Brasilia, v. 61, n. 2, p. 209-214, mar./abr. 2008.

KOLLER, M. Health risks related to shitwork: na example of time-contingent effects of long-term stress. Archive of Occupational and Environmental Health, New York, v. 53, p. 59-75, 1983.

MENDES, R. Patotogias do trabalho. Rio de Janeiro: Atheneu, 1995.

MORENO, C. R. C.; FISCHER, F. M.; ROTENBERG, L. A saúde do trabalhador na sociedade 24 horas. São Paulo Perspectiva, São Paulo, v. 17, n. 1, p. 43-48, jan./ mar. 2003.

NAVARRO, V. L.; PADILHA, V. Dilemas do trabalho no capitalismo contemporâneo. Psicologia \& Sociedade, Porto Alegre, v. 19, n. esp., p.14-20, 2007.

OTERO, J. J. G. Riesgos del trabajo del personal sanitario. 2. ed. Madrid: McGRAW-HILL-Interamericana, 1993.

PARRA, M. T. Ações administrativas dos enfermeiros, frente ao absenteísmo na enfermagem em um hospital universitário. 2003. Dissertação (Mestrado em Enfermagem Fundamental) - Escola de Enfermagem de Ribeirão Preto, Universidade de São Paulo, Ribeirão 
Preto.

PITTA, A.M. F. Hospital: dor e morte como ofício. 5. ed. São Paulo: Annablume/ Hucitec, 2003.

QUICK, T. C.; LAPERTOSA, J. B. Análise do absenteísmo em usina siderúrgica. Revista Brasileira de Saúde Ocupacional, São Paulo, v. 18, n. 69, p. 65-70, 1982.

REIS, R. J.; LA ROCC, P. de F.; SILVEIRA, A. M.; BONILLA, I. M. L.; GINÉ, A. N.; MARTÍN, M. Fatores relacionados ao absenteísmo por doença em profissionais de enfermagem. Revista de Saúde Pública, São Paulo, v. 37, n. 5, p. 616-623, 2003.

ROBBINS, S. P. Comportamento organizacional. 9. ed. São Paulo: Prentice Hall, 2005.

RODRIGUES, C. C. M. Introdução à saúde ocupacional. Londrina: Instituto Brasileiro de Estudos e Pesquisa Sócio-Econômicas, 2004. Apostila do Curso de Especialização em Enfermagem do Trabalho.

ROTEMBERG, L.; PORTELA, L. F; MARCONDES, W. B.; MORENO, C. R. C.; NASCIMENTO, C. P. Gênero e trabalho noturno: sono, cotidiano e vivências de quem troca a noite pelo dia. Cadernos de Saúde Pública, v. 17, p. 639-649, 2001.

SILVA, D. M. P. P.; MARZIALE, M. H. P. Absenteísmo de trabalhadores de enfermagem em um hospital universitário. Revista Latino-Americana de Enfermagem, Ribeirão Preto, v. 8, n. 5, p. 44-51, out. 2000.

Problemas de saúde responsáveis pelo absenteísmo de trabalhadores de enfermagem de um hospital universitário. Acta Scientiarum: Health Sciences, Maringá, v. 25, n. 2, p. 191-197, 2003.

Recebido em 26 de março de 2010 - Received on March 26, 2010

Aceito em 17 de janeiro de 2011 - Accepted on January 17, 2011 


\section{Apêndice A}

Instrumento de coleta de dados - Absenteísmo

1. $\quad \mathrm{N}^{\circ}$ do formulário:

2. Gerência: ( 1 ) Adm. ( 2 ) Enf. 3. Sexo: ( 1 ) Fem. ( 2 ) Masc.

4. Data de Nascimento:

Idade

5. Data de Admissão:

Tempo de serviço

6. Categoria funcional:

7. Jornada de trabalho semanal (em horas): ( 1 ) 20

( 2 ) 36

(3) 42

(4) 44

8. Turno de trabalho: ( 1 ) Manhã ( 2 ) Tarde ( 3 ) Manhã/Tarde ( 4 ) Integral ( 4 ) Noite

\begin{tabular}{|c|c|c|c|c|c|c|c|}
\hline \multirow[t]{2}{*}{ Mês / Ano } & \multirow{2}{*}{$\begin{array}{c}\text { Especialidade } \\
\text { Profissional }\end{array}$} & \multicolumn{6}{|c|}{ Dias de Afastamento } \\
\hline & & 1 & 2 & 3 & 4 a 15 & $16 /+$ & CID \\
\hline & & & & & & & \\
\hline & & & & & & & \\
\hline & & & & & & & \\
\hline & & & & & & & \\
\hline & & & & & & & \\
\hline & & & & & & & \\
\hline & & & & & & & \\
\hline
\end{tabular}

